Article

\title{
Some New Extensions on Fractional Differential and Integral Properties for Mittag-Leffler Confluent Hypergeometric Function
}

\author{
F. Ghanim ${ }^{1, *,+} \mathbb{D}$, Hiba F. Al-Janaby ${ }^{2,+}$ and Omar Bazighifan $3,4, *,+\mathbb{D}$ \\ 1 Department of Mathematics, College of Sciences, University of Sharjah, Sharjah 27272, United Arab Emirates \\ 2 Department of Mathematics, College of Sciences, University of Baghdad, Baghdad 10081, Iraq; \\ fawzihiba@yahoo.com \\ 3 Section of Mathematics, International Telematic University Uninettuno, Corso Vittorio Emanuele II, 39, \\ 00186 Rome, Italy \\ 4 Department of Mathematics, Faculty of Science, Hadhramout University, Hadhramout 50512, Yemen \\ * Correspondence: fgahmed@sharjah.ac.ae (F.G.); o.bazighifan@gmail.com (O.B.) \\ + These authors contributed equally to this work.
}

check for

updates

Citation: Ghanim, F.; Al-Janaby, H.F.; Bazighifan, O. Some New Extensions on Fractional Differential and Integral Properties for Mittag-Leffler Confluent Hypergeometric Function. Fractal Fract. 2021, 5, 143. https:// doi.org/10.3390/fractalfract5040143

Academic Editor: Enzo Orsingher

Received: 6 September 2021

Accepted: 24 September 2021

Published: 29 September 2021

Publisher's Note: MDPI stays neutral with regard to jurisdictional claims in published maps and institutional affiliations.

Copyright: (c) 2021 by the authors. Licensee MDPI, Basel, Switzerland. This article is an open access article distributed under the terms and conditions of the Creative Commons Attribution (CC BY) license (https:// creativecommons.org/licenses/by/ $4.0 /)$

\begin{abstract}
This article uses fractional calculus to create novel links between the well-known MittagLeffler functions of one, two, three, and four parameters. Hence, this paper studies several new analytical properties using fractional integration and differentiation for the Mittag-Leffler function formulated by confluent hypergeometric functions. We construct a four-parameter integral expression in terms of one-parameter. The paper explains the significance and applications of each of the four Mittag-Leffler functions, with the goal of using our findings to make analyzing specific kinds of experimental results considerably simpler.
\end{abstract}

Keywords: mittag-leffler function; laplace transform; confluent hypergeometric function; fractional calculus; integral operator

\section{Introduction}

The regular integration and differentiation in calculus operations are extended to instructions beyond the integers in fractional calculus: to determine the order of differentiation, real and complex numbers may be used [1-3]. This topic is more than 400 years old, although it has been greatly developed over the previous century, uncovering applications in a wide range of scientific and engineering areas [4-6].

Riemann-Liouville is the most important concept of fractional derivatives and integrals, where the meanings are provided by the following definitions:

$$
{ }_{a}^{R L} D_{x}^{\alpha} f(x)=\frac{1}{\Gamma(-\alpha)} \int_{a}^{x}(x-t)^{-\alpha-1} f(t) d t, \quad \Re(\alpha)<0,
$$

and

$$
{ }_{a}^{R L} D_{x}^{\alpha} f(x)=\frac{d^{m}}{d x^{m}}\left({ }_{a}^{R L} D_{x}^{\alpha-m} f(x)\right), \quad \Re(\alpha) \geq 0, m:=[\Re(\alpha)]+1,
$$

where $D^{\alpha} f$ refers to the derivative of order $\alpha$ for the function $f$, and $a$ is the diffintegration constant.

It is essential to keep in mind that derivatives and integrals in fractional calculus are detected by the use of an arbitrary constant $a$. The value of $a$ is usually set to one of two values: $a=0$ or $a=-\infty$. We establish the following lemma's validity as a source of two "natural" differintegration formulae, $a=0$ and $a=-\infty$; both are viable choices to understand the advantages of both choices. Neither option may be excluded from the set of potential values for $a$. 
Lemma 1 ([7]). With differintegration constants $a=0$ and $a=-\infty$, the Riemann-Liouville (RL) differintegrals of exponential and power functions are as follows:

$$
\begin{gathered}
{ }_{0}^{R L} D_{x}^{\alpha} f\left(x^{\gamma}\right)={ }^{R L} D_{x}^{\alpha} f\left(x^{\gamma}\right)=\frac{\Gamma(\gamma+1)}{\Gamma(\gamma-\alpha+1)} x^{\gamma-\alpha}, \quad \alpha, \gamma \in \mathbb{C}, \Re(\gamma)>-1 ; \\
{ }_{-\infty}^{R L} D_{x}^{\alpha}\left(e^{\gamma x}\right)=\gamma^{\alpha} e^{\gamma x}, \quad \alpha, \gamma \in \mathbb{C}, \gamma \notin \mathbb{R}_{0}^{-} .
\end{gathered}
$$

To describe complex power functions, we utilize the main branch with values ranging from - pi to pi in both equations; also see Reference [1-3].

Many recent definitions and investigations of fractional differintegrals have been considered recently. Part of them was aided by a number of well recognized systems that can be formed by various structures based on fractional calculus. As an example to better demonstrate certain processes in dynamical systems, in Equation (1), another function is used in lieu of the power function [8,9]. Other processes were created by integrating more generalization levels and parameters into formulas and functions [10-12].

The Mittag-Leffler function is a precise function that appears often in the study of fractional integrals and derivatives; see, for example, Ghanim and Al-Janaby [13,14], Ghanim et al. [15], Oros [16,17], Haubold et al. [18], Paneva-Konovska [19], Mainardi and Gorenflo [20], Mathai and Haubold [21], Srivastava [22,23], and Srivastava et al. [24].

In 1903, Magnus Gustaf (Gösta) Mittag-Leffler (1846-1927) [25] (also see MittagLeffler [26]), a Swedish mathematician, invented and studied the well-known Mittag-Leffler function $E_{\alpha}(z)$ given by

$$
E_{\alpha}(z)=\sum_{n=0}^{\infty} \frac{z^{n}}{\Gamma(\alpha n+1)} \quad(z \in \mathbb{C} ; \Re(\alpha)>0) .
$$

Wiman [27] and Reference [28] then proposed a generalization $E_{\alpha}(z)$ of $E_{\alpha, \gamma}(z)$ provided by

$$
E_{\alpha, \gamma}(z)=\sum_{n=0}^{\infty} \frac{z^{n}}{\Gamma(\alpha n+\gamma)} \quad(\Re(\alpha)>0 ; \gamma, \alpha \in \mathbb{C}) .
$$

As a result, the functions of Mittag-Leffler $E_{\alpha}(z)$ and $E_{\alpha, \gamma}(z)$ in (5) and (6), respectively, have been studied and expanded in a variety of different ways and applications. The implementation in the physical model has succeeded in recent decades, the generalized Mittag-Leffler functions were also used in mathematical and physical issues, as the solutions of the fractional integral and differential equations were naturally presented. Fractional order calculus is associated with practical endeavors, and it is widely used in nanotechnology [29], chaos theory [30], optics [31], human diseases [32], and other fields $[33,34]$. In fact, the authors are collaborating with a group of college of engineering researchers on several recent engineering applications involving generalized multi-parameter Mittag-Leffler functions and their extended types, such as noise measurement and heat transfer in asphalt concrete. Wright [35] investigated these functions and their associated disseminations. Pillai [36] also established links between generalized Mittag-Leffler type functions and route models. Ref. [37,38] are two studies which have utilized the function (5) with parameters $\alpha$ and $\gamma$ in more general functions linked to one or more parameters.

$$
E_{\alpha, \gamma}^{\lambda}(z)=\sum_{n=0}^{\infty} \frac{\Gamma(n+\lambda) z^{n}}{\Gamma(\lambda) \Gamma(\alpha n+\gamma) n !}, \quad(\Re(\alpha)>0 ; \lambda, \gamma, \alpha \in \mathbb{C}) .
$$




$$
E_{\alpha, \gamma}^{\beta, \lambda}(z)=\sum_{n=0}^{\infty} \frac{\Gamma(\beta n+\lambda) z^{n}}{\Gamma(\lambda) \Gamma(\alpha n+\gamma) n !}, \quad(\Re(\alpha)>0 ; \lambda, \gamma, \alpha \in \mathbb{C}) .
$$

A variety of physical disciplines have shown the application for the functions of confluent hypergeometric. It has been employed in cases where both sedileritition and diffusion are related; in ultracentrifuges, for example, isotope separation and protein molecular weight measurements are applications. Such functions are often used to express the formula for the velocity distribution of electrons in high-frequency gas discharges.

The solution of the second-order linear homogeneous differential equation for the confluent hypergeometric function $M(\lambda ; \gamma ; z)$ is as follows:

$$
z \frac{d^{2} M}{d z^{2}}+(\gamma-z) \frac{d M}{d z}-\lambda M=0, \quad(\lambda, \gamma, z \in \mathbb{C}) .
$$

Equation (9) contains a sporadic singularity at infinity, as well as a normal initial singularity [39].

A second solution of Equation (9) may be found if $\gamma$ is not integral.

$$
W(\lambda ; \gamma ; z)=z^{1-\gamma} M(\lambda-\gamma+1 ; 2-\gamma ; z) .
$$

If $\gamma$ is integral, then

$$
\begin{aligned}
W(\alpha ; \gamma ; z)= & M(\alpha ; \gamma ; z)\{\ln z+\Omega(1-\alpha)-\Omega(\gamma)+C\}+\sum_{n=1}^{\infty} \frac{\Gamma(n+\lambda) \Gamma(\gamma) B_{n} z^{n}}{\Gamma(\lambda) \Gamma(n+\gamma) n !} \\
& +(-1)^{\gamma} \sum_{n=0}^{\infty} \frac{\Gamma(\gamma) \Gamma(n+\lambda-\gamma+1) \Gamma(\gamma-n-1)(-1)^{n}}{\Gamma(\lambda) n ! z}
\end{aligned}
$$

may be used to provide a second solution, where

$$
\Omega(\lambda)=\frac{\Gamma^{\prime}(\lambda)}{\mathrm{T}(\lambda)}
$$

where the Euler's constant $C$ is equal to $0.577216 \ldots$, and

$$
\begin{gathered}
B_{n}=\left(\frac{1}{\lambda}+\frac{1}{\lambda+1}+\ldots+\frac{1}{\lambda+n-1}\right) \\
-\left(\frac{1}{\gamma}+\frac{1}{\gamma+1}+\ldots+\frac{1}{\gamma+n-1}\right)-\left(1+\frac{1}{2}+\ldots+\frac{1}{n}\right) .
\end{gathered}
$$

The $\Omega$ function's elaboration tables are given in Reference [40].

Furthermore, $M(\lambda ; \gamma ; z)$ has a series of representation in

$$
M(\alpha ; \gamma ; z)=1+\frac{\lambda}{\gamma} z+\frac{\lambda(\lambda+1)}{\gamma(\gamma+1)} \frac{z^{2}}{2}+\ldots=\sum_{n=0}^{\infty} \frac{\Gamma(\gamma) \Gamma(n+\lambda) z^{n}}{\Gamma(\lambda) \Gamma(n+\gamma) n !} .
$$

For all values of $z$, it is obvious that the previous series absolutely converges. The following are guaranteed by both $M$ and $W$ :

$$
\begin{gathered}
\frac{d}{d z} M(\lambda ; \gamma ; z)=\frac{\lambda}{\gamma} M(\lambda+1 ; \gamma+1 ; z), \\
\lambda M(\lambda+1 ; \gamma+1 ; z)=(\lambda-\gamma) M(\lambda ; \gamma+1 ; z)+\gamma M(\lambda ; \gamma ; z), \\
\lambda M(\lambda+1 ; \gamma ; z)=(z+2 \lambda-\gamma) M(\lambda ; \gamma ; z)+(\gamma-\lambda) M(\lambda-1 ; \gamma ; z) .
\end{gathered}
$$


Equation (13) follows directly, since the differentiation of Equations (12), (14) and (15) may be obtained subsequently from the differential equation. Using the Wronskian of Equation (9), the functional connection between a Wronskian's value at any point in a plane and its value at a specific place may be utilized to demonstrate a relationship between $M(\lambda ; \gamma ; z)$ and $W(\alpha ; \gamma ; z)$. For additional information, see References [39-41].

The confluent hypergeometric Mittag-Leffler function $M_{\alpha, \gamma}^{\beta, \lambda}(z)$ is now presented as follows:

$$
M_{\alpha, \gamma}^{\beta, \lambda}(z)=\sum_{n=0}^{\infty} \frac{\Gamma(\gamma) \Gamma(\beta n+\lambda) z^{n}}{\Gamma(\lambda) \Gamma(\alpha n+\gamma) n !}, \quad(\alpha, \gamma, \beta, \lambda \in C ; \Re(\alpha)>0) .
$$

The following are some special instances of the confluent hypergeometric MittagLeffler function $M_{\alpha, \gamma}^{\beta, \lambda}(z)$ :

$$
\begin{gathered}
M_{0, \gamma}^{0, \lambda}(z)=e^{z}, \quad M_{0,1}^{1,1}(z)=\frac{1}{1-z^{\prime}}, \quad M_{1,2}^{1,1}(z)=\frac{e^{z}-1}{z}, \\
M_{2,1}^{1,1}\left(z^{2}\right)=\cosh z, \quad M_{2,1}^{1,1}\left(-z^{2}\right)=\cos z \\
M_{1, \gamma}^{1, \lambda}(z)=M(\alpha ; \gamma ; z) \\
M_{\alpha, 1}^{1,1}(z)=E_{\alpha}(z)=\sum_{n=0}^{\infty} \frac{z^{n}}{\Gamma(\alpha n+1)} .
\end{gathered}
$$

When $\alpha$ is a positive integer, such as $n$, then:

$$
M_{\alpha, \gamma}^{1, \lambda}(z)={ }_{1} F_{n}\left(\lambda ; \frac{\gamma}{n}, \frac{\gamma+1}{n}, \ldots, \frac{\gamma+n-1}{n} ; \frac{z}{n^{n}}\right) ;
$$

in addition,

$$
\begin{gathered}
H_{i}(x, n)=x^{i-1} M_{n, i}^{1,1}\left(x^{n}\right), \\
T_{i}(x, n)=x^{i-1} M_{n, i}^{1,1}\left(-x^{n}\right) .
\end{gathered}
$$

$H_{i}$ and $T_{i}$ are generalized hyperbolic and trigonometrical functions, respectively [42]. Furthermore,

$$
\begin{gathered}
\left(\frac{d}{d z}\right)^{m} M_{\alpha, \gamma}^{\beta, \lambda}(z)=(\lambda)_{m} M_{\alpha, \gamma+m}^{\beta, \lambda+m}(z), \\
\left(\frac{d}{d z}\right)^{m} z^{\gamma-1} M_{\alpha, \gamma}^{\beta, \lambda}\left(z^{\alpha}\right)=z^{\gamma-m-1} M_{\alpha, \gamma}^{\beta, \lambda}\left(z^{\alpha}\right), \\
\left(z \frac{d}{d z}+\lambda\right) M_{\alpha, \gamma}^{\beta, \lambda}\left(z^{\alpha}\right)=\lambda M_{\alpha, \gamma}^{\beta, \lambda+1}(z), \\
(\gamma-\alpha \lambda-1) M_{\alpha, \gamma}^{\beta, \lambda}(z)=M_{\alpha, \gamma-1}^{\beta, \lambda}(z)-\alpha \lambda M_{\alpha, \gamma}^{\beta, \lambda+1}(z),
\end{gathered}
$$


and

$$
\mathcal{L}\left\{t^{\gamma-1} M_{\alpha, \gamma}^{\beta, \lambda}\left(\mu t^{\alpha}\right)\right\}=\rho^{-\gamma}\left(1-\mu \rho^{-\alpha}\right)^{-\lambda} \quad|\rho|>|\mu|^{\frac{1}{\Re(\alpha)}}, \Re(\rho)>0, \Re(\gamma)>0 .
$$

In this case, the Laplace transform of $f(t)$ is represented by $\mathcal{L}\{f(t)\}$.

Several scholars (see, for example, References [43-48]) have utilized the Laplace transform to solve convolution equations, which are special instances of

$$
\int_{0}^{x} \frac{(x-t)^{b-1}}{\Gamma(b)}{ }_{1} F_{1}(a ; b ; c(x-t)) f(t) d t=g(x) \quad \Re(b)>0,
$$

described in fractional integration [49]. The target of this research is to look into an integral equation

$$
\int_{a}^{x}(t-x)^{\gamma-1} M_{\alpha, \gamma}^{\beta, \lambda} \mu(t-x)^{\alpha} f(t) d t=g(x) \quad \Re(\gamma)>0,
$$

for any real number $a>0$, where the function $M_{\alpha, \gamma}^{\beta, \lambda}(z)$ in (16) is an analytic function of order $\alpha$ that includes many well-known unique functions.

Ghanim and Al-Janaby [50] introduced (23) by using an integral operator $\Sigma_{\alpha, \gamma}^{\beta, \lambda}(\mu)$ on a space $\Psi$ of functions and a fractional integration operator $\Delta^{\delta}: \Psi \rightarrow \Psi$ to prove results on $\Sigma_{\alpha, \gamma}^{\beta, \lambda}(\mu)$. Theorems on the solutions of (23) were then discussed using these results. This technique may be used to obtain comparable results on the integral equation

$$
\mathfrak{E}_{\alpha, \gamma}^{\beta, \lambda}(\mu) f(x) \equiv \int_{a}^{x}(t-x)^{\gamma-1} M_{\alpha, \gamma}^{\beta, \lambda} \delta(t-x)^{\alpha} f(t) d t=g(x) \quad \Re(\gamma)>0,
$$

which consists of the equations given in References [51,52] as special cases. The linear space of complex-valued functions $f$ that are $\Psi$-integrable on a finite $[a, b], a \geq 0$ with $\|f\|=\int_{a}^{b}|f(t)| d t$ is denoted by $\Psi . \Delta^{\delta}: \Psi \rightarrow \Psi$ is a fractional operator defined by the fractional integral for complex $\delta$ with $\Re(\delta)>0$

$$
\Delta^{\delta} f(x)=\int_{a}^{x} \frac{(x-t)^{\delta-1}}{\Gamma(\delta)} f(t) d t
$$

The fact that $\Delta^{\delta}$ is bounded and that $\Delta^{\delta} f=0 \rightarrow f=0$ is a standard result means that the inverse operator exists on the $\Psi_{\delta}$ subspace of $\Psi$. If $0<\Re(\delta)<\Re(\xi)$, it is easily demonstrated that $\Psi_{\xi} \subset \Psi_{\delta} \subset \Psi$, and the inclusion is appropriate. For $\Re(\delta)<0, \Delta^{\delta}$, the inverse of $\Delta^{-\delta}$ is defined. If $\Re(\delta) \neq 0$ and $\Re(\xi) \neq 0$, then $\Delta^{\delta} \Delta^{\xi} f=\Delta^{\delta+\xi}$ for appropriate functions $f$. $\Delta^{\delta}$ is defined on $\Psi_{\mu}$ with $\Re(\mu)>0$ as $\Delta^{-1} \Delta^{1+\delta}$ for $\Re(\delta)=0$. For complex $\alpha, \gamma, \lambda, \mu$ with $f \in \Psi$ and $\Re(\gamma)>0$, the fractional operator $\Sigma_{\alpha, \gamma}^{\beta, \lambda}$ on $\Psi$ into itself is defined by

$$
\Sigma_{\alpha, \gamma}^{\beta, \lambda}(\mu) f(x)=\int_{a}^{x}(t-x)^{\gamma-1} M_{\alpha, \gamma}^{\beta, \lambda} \mu(t-x)^{\alpha} f(t) d t \quad a<x<b .
$$

Lemma 2 ([53]). Assume $f$ is a function defined by

$$
f(x)=\sum_{n=1}^{\infty} f_{n}(x)
$$

that uniformly converges on $|x-a| \leqslant L$, where $L>0$ and $a \in \mathbb{C}$ are fixed constants. For a fixed order of differintegration $\alpha \in \mathbb{C}$, if 1 . $\Re(\alpha)<0$ (fractional integration), then

$$
{ }_{a}^{R L} D_{x}^{\alpha} f(x)=\sum_{n=1}^{\infty}{ }_{a}^{R L} D_{x}^{\alpha} f_{n}(x), \quad|x-a| \leqslant L,
$$


and the right side of the series is uniformly convergent on $|x-a| \leqslant L$.

2. $\Re(\alpha) \geqslant 0$ (fractional differentiation) and $\sum_{n=1}^{\infty}{ }_{a}^{R L} D_{x}^{\alpha} f_{n}(x)$ is uniformly convergent series on the given region, then

$$
{ }_{a}^{R L} D_{x}^{\alpha} f(x)=\sum_{n=1}^{\infty}{ }_{a}^{R L} D_{x}^{\alpha} f_{n}(x), \quad|x-a| \leqslant L .
$$

\section{Results}

Theorem 1. Let $\alpha, \beta, \lambda, \gamma \in \mathbb{C}$ with $\Re(\alpha), \Re(\gamma)>0$; then:

$$
M_{\alpha, \gamma}^{\beta, \lambda}(z)=\frac{\Gamma(\gamma)}{\Gamma(\lambda)}{ }^{0} D_{z}^{n \beta+\lambda-n-1}\left[z^{\lambda-1} E_{\alpha, \gamma}\left(z^{\beta}\right)\right], \quad z \in \mathbb{C} .
$$

Proof. It is clear from Lemma 1 that most often a quotient of gamma functions can be defined as originating from a fractional power function differintegral. The expression $\frac{\Gamma(\beta n+\lambda)}{n !}$ in the coefficients of the series Equation (16) yields the following:

$$
\begin{gathered}
M_{\alpha, \gamma}^{\beta, \lambda}(z)=\sum_{n=0}^{\infty} \frac{\Gamma(\gamma) \Gamma(\beta n+\lambda) z^{n}}{\Gamma(\lambda) \Gamma(\alpha n+\gamma) n !}=\sum_{n=0}^{\infty} \frac{\Gamma(\gamma)}{\Gamma(\lambda) \Gamma(\alpha n+\gamma)} \frac{\Gamma(\beta n+\lambda)}{\Gamma(n+1)} z^{n} \\
=\sum_{n=0}^{\infty} \frac{\Gamma(\gamma)}{\Gamma(\lambda) \Gamma(\alpha n+\gamma)}{ }^{R L} D_{z}^{n \beta+\lambda-n-1}\left[z^{n \beta+\lambda-1}\right] \\
=\sum_{n=0}^{\infty} \frac{\Gamma(\gamma)}{\Gamma(\lambda){ }^{R}}{ }^{R L} D_{z}^{n \beta+\lambda-n-1}\left[\frac{z^{n \beta+\lambda-1}}{\Gamma(\alpha n+\gamma)}\right] .
\end{gathered}
$$

We may utilize the Lemma 2 result to replace the summation with fractional differential integration since the series is uniformly convergent. Then, we obtain:

$$
\begin{gathered}
M_{\alpha, \gamma}^{\beta, \lambda}(z)=\frac{\Gamma(\gamma)}{\Gamma(\lambda)} \sum_{n=0}^{\infty}{ }_{0}^{R L} D_{z}^{n \beta+\lambda-n-1}\left[\frac{z^{n \beta+\lambda-1}}{\Gamma(\alpha n+\gamma)}\right] \\
=\frac{\Gamma(\gamma)}{\Gamma(\lambda)}{ }^{R L} D_{z}^{n \beta+\lambda-n-1}\left[\sum_{n=0}^{\infty} \frac{z^{n \beta+\lambda-1}}{\Gamma(\alpha n+\gamma)}\right] \\
=\frac{\Gamma(\gamma)}{\Gamma(\lambda)^{0}}{ }^{R L} D_{z}^{n \beta+\lambda-n-1}\left[z^{\lambda-1} \sum_{n=0}^{\infty} \frac{z^{n \beta}}{\Gamma(\alpha n+\gamma)}\right] \\
=\frac{\Gamma(\gamma)}{\Gamma(\lambda)}{ }^{0}{ }^{R L} D_{z}^{n \beta+\lambda-n-1}\left[z^{\lambda-1} E_{\alpha, \gamma}\left(z^{\beta}\right)\right] .
\end{gathered}
$$

Hence, we have the result.

Corollary 1. For any $\alpha, \lambda \in \mathbb{C}$ with $\Re(\alpha)>0$, we have:

$$
M_{\alpha}^{\beta, \lambda}(z)=\frac{\Gamma(\gamma)^{R L}}{\Gamma(\lambda)^{0}} D_{z}^{n \beta+\lambda-n-1}\left[z^{\lambda-1} E_{\alpha}\left(z^{\beta}\right)\right] .
$$

Proof. The proof is immediately followed by replacing $\gamma$ with 1 in Theorem 1 . 
It is obvious that, if we recover the basic identity, $M_{\alpha, 1}^{1,1}(z)=E_{\alpha}(z)=\sum_{n=0}^{\infty} \frac{z^{n}}{\Gamma(\alpha n+1)}$ by establishing $\beta=\gamma=\lambda=1$ in Equation (26). Using the exact same logic as in Theorem 1, we can demonstrate this.

Corollary 2. Let $\alpha, \beta, \lambda, \gamma, \tau \in \mathbb{C}$ with $\Re(\alpha)>0, \Re(\gamma)>0$. We obtain:

$$
M_{\alpha, \gamma}^{\beta, \lambda}(\tau z)=\frac{\Gamma(\gamma)}{\Gamma(\lambda)^{0}}{ }^{R L} D_{z}^{n \beta+\lambda-n-1}\left[z^{\lambda-1} E_{\alpha, \gamma}\left(\tau z^{\beta}\right)\right], \quad z \in \mathbb{C} .
$$

Proof. The proof is as proven above in Theorem 1 with an additional element of $\tau^{n}$ contained in each of the sum terms.

Theorem 2. For $\alpha, \beta, \gamma, \tau \in \mathbb{C}$ and $\lambda=1$ with $\Re(\alpha)>0, \Re(\gamma)>0$, we have:

$$
M_{\alpha, \gamma}^{\beta}\left(\tau z^{\alpha}\right)=\frac{z^{1-\gamma}}{\Gamma(\gamma)}{ }^{R}{ }^{0} D_{z}^{n \beta-\alpha n-\gamma+1}\left[E_{\alpha, \gamma}\left(\tau z^{\alpha \beta}\right)\right], \quad z \in \mathbb{C} .
$$

Proof. We start this time from the right side of the appropriate identity and then use the definition in Equation (6) of the function $E_{\alpha, \gamma}$ :

$$
\begin{gathered}
\frac{z^{1-\gamma}}{\Gamma(\gamma)}{ }^{R} D_{z}^{n \beta-\alpha n-\gamma+1}\left[E_{\alpha, \gamma}\left(\tau z^{\alpha \beta}\right)\right]=\frac{z^{1-\gamma}}{\Gamma(\gamma)}{ }^{0} D_{z}^{n \beta-\alpha n-\gamma+1}\left[\sum_{n=0}^{\infty} \frac{\left(\tau z^{\alpha \beta}\right)^{n}}{\Gamma(\alpha n+\gamma)}\right] \\
=\frac{z^{1-\gamma}}{\Gamma(\gamma)}{ }^{R}{ }^{0} D_{z}^{n \beta-\alpha n-\gamma+1}\left[\sum_{n=0}^{\infty} \frac{\tau^{n} z^{n \alpha \beta}}{\Gamma(\alpha n+\gamma)}\right] .
\end{gathered}
$$

The series is uniformly convergent, so we can switch the summation and fractional differintegration by Lemma 2 given that the resulting series always converges uniformly (at least in the case of $1>\Re(\gamma)>0$ ). Then, we switch the operations and end with the appropriate illustration.

$$
\begin{aligned}
& \frac{z^{1-\gamma}}{\Gamma(\gamma)}{ }^{0}{ }^{R} D_{z}^{n \beta-\alpha n-\gamma+1}\left[E_{\alpha, \gamma}\left(\tau z^{\alpha \beta}\right)\right]=\frac{z^{1-\gamma}}{\Gamma(\gamma)} \sum_{n=0}^{\infty}{ }_{0}^{R L} D_{z}^{n \beta-\alpha n-\gamma+1}\left[\frac{\tau^{n} z^{n \alpha \beta}}{\Gamma(\alpha n+\gamma)}\right] \\
& =\frac{z^{1-\gamma}}{\Gamma(\gamma)} \sum_{n=0}^{\infty} \frac{\Gamma(\beta n+1)}{\Gamma(\alpha n+\gamma)} \frac{\tau^{n} z^{n \alpha \beta}}{\Gamma(\beta n+1)}=\sum_{n=0}^{\infty} \frac{\tau^{n} z^{n \alpha \beta}}{\Gamma(\beta n+1)} .
\end{aligned}
$$

The series converges uniformly, being explicitly in the Equation (6) series expression for $E_{\alpha, \gamma}\left(\tau z^{\beta}\right)$. Thus, our aforementioned switching of operations was appropriate, and the proof is now true.

Corollary 3. For $\alpha, \beta, \tau \in \mathbb{C}$ and $\gamma=\lambda=1$ with $\Re(\alpha)>0$, we have:

$$
M_{\alpha}^{\beta}(\tau z)=z_{0}^{1-\gamma R L} D_{z}^{n(\beta-\alpha)}\left[E_{\alpha}\left(\tau z^{\alpha \beta}\right)\right] .
$$

Proof. The proof is immediately followed by replacing $\gamma$ with 1 in Theorem 2 .

By combining Theorems 1 and 2, a composite expression can be obtained in Equation (6) for the four-parameter Mittag-Leffler function, as defined in the following theorem, in terms of fractional integrals. 
Theorem 3. Let $\alpha, \beta, \lambda, \gamma, \tau \in \mathbb{C}$ and $\Re(\alpha)>0, \Re(\gamma)>0$ with $\Re(\lambda)<1$, and we obtain:

$$
\begin{gathered}
M_{\alpha, \gamma}^{\beta, \lambda}\left(\tau z^{\alpha}\right)=\frac{\alpha \sin (\pi \lambda) B(1-\lambda, n-n \beta)}{\pi \Gamma(n-n \beta)} \\
\times \int_{0}^{z}\left(z^{\alpha}-u^{\alpha}\right)^{-n \beta+n-\lambda} u^{\alpha(n \beta+\lambda-n)-\gamma R L} D_{u}^{n \beta-\alpha n-\gamma+1}\left[E_{\alpha, \gamma}\left(\tau u^{\alpha \beta}\right)\right] d u,
\end{gathered}
$$

where $z \in \mathbb{C}$ and $B$ is beta function.

Proof. For $\Re(\lambda)<1$, the fractional differintegrals showing up in Theorem 1 and Corollary 1 are integrals, so Equation (28) can be modified as follows:

$$
\begin{gathered}
M_{\alpha, \gamma}^{\beta, \lambda}(\tau z)=\frac{\Gamma(\gamma)}{\Gamma(\lambda)}{ }^{0}{ }^{0} D_{z}^{n \beta+\lambda-n-1}\left[z^{\lambda-1} E_{\alpha, \gamma}\left(\tau z^{\beta}\right)\right] \\
=\frac{\Gamma(\gamma)}{\Gamma(\lambda) \Gamma(n+1-n \beta-\lambda)} \int_{0}^{z}(z-y)^{-n \beta+n-\lambda} y^{n \beta+\lambda-n-1}\left[E_{\alpha, \gamma}\left(\tau y^{\beta}\right)\right] d y .
\end{gathered}
$$

Then, we shift variables $y$ with $u^{\alpha}$ and use the reflection formula for the gamma function with the definition of beta function to have

$$
\begin{gathered}
M_{\alpha, \gamma}^{\beta, \lambda}(\tau z)=\frac{\Gamma(\gamma) \sin (\pi \lambda) B(1-\lambda, n-n \beta)}{\pi \Gamma(n-n \beta)} \\
\times \int_{0}^{z^{1 / \alpha}}\left(z-u^{\alpha}\right)^{-n \beta+n-\lambda} u^{\alpha(n \beta+\lambda-n-1)}\left[E_{\alpha, \gamma}\left(\tau u^{\alpha \beta}\right)\right] \alpha u^{\alpha-1} d u \\
=\frac{\alpha \Gamma(\gamma) \sin (\pi \lambda) B(1-\lambda, n-n \beta)}{\pi \Gamma(n-n \beta)} \\
\times \int_{0}^{z^{1 / \alpha}}\left(z-u^{\alpha}\right)^{-n \beta+n-\lambda} u^{\alpha(n \beta+\lambda-n)-1}\left[E_{\alpha, \gamma}\left(\tau u^{\alpha \beta}\right)\right] d u .
\end{gathered}
$$

Applying the result of Theorem 2, we have

$$
\begin{aligned}
& M_{\alpha, \gamma}^{\beta, \lambda}(\tau z)=\frac{\alpha \Gamma(\gamma) \sin (\pi \lambda) B(1-\lambda, n-n \beta)}{\pi \Gamma(n-n \beta)} \\
& \times \int_{0}^{z^{1 / \alpha}}\left(z-u^{\alpha}\right)^{-n \beta+n-\lambda} u^{\alpha(n \beta+\lambda-n)-1}\left[\frac{u^{1-\gamma}}{\Gamma(\gamma)}{ }^{0} L D_{z}^{n \beta-\alpha n-\gamma+1}\left[E_{\alpha, \gamma}\left(\tau u^{\alpha \beta}\right)\right]\right] d u \\
& =\frac{\alpha \sin (\pi \lambda) B(1-\lambda, n-n \beta)}{\pi \Gamma(n-n \beta)} \\
& \times \int_{0}^{z^{1 / \alpha}}\left(z-u^{\alpha}\right)^{-n \beta+n-\lambda} u^{\alpha(n \beta+\lambda-n)-\gamma R L} D_{z}^{n \beta-\alpha n-\gamma+1}\left[E_{\alpha, \gamma}\left(\tau u^{\alpha \beta}\right)\right] d u .
\end{aligned}
$$

Substituting $z^{\alpha}$ for $z$, we obtain:

$$
M_{\alpha, \gamma}^{\beta, \lambda}\left(\tau z^{\alpha}\right)=\frac{\alpha \sin (\pi \lambda) B(1-\lambda, n-n \beta)}{\pi \Gamma(n-n \beta)}
$$




$$
\times \int_{0}^{z}\left(z^{\alpha}-u^{\alpha}\right)^{-n \beta+n-\lambda} u^{\alpha(n \beta+\lambda-n)-\gamma R L} D_{u}^{n \beta-\alpha n-\gamma+1}\left[E_{\alpha, \gamma}\left(\tau u^{\alpha \beta}\right)\right] d u .
$$

Hence, we have the result.

Corollary 4. Let $\alpha, \beta, \lambda, \tau \in \mathbb{C}$ and $\Re(\alpha)>0$ with $\Re(\lambda)<1$, and we have:

$$
\begin{gathered}
M_{\alpha}^{\beta, \lambda}\left(\tau z^{\alpha}\right)=\frac{\alpha \sin (\pi \lambda) B(1-\lambda, n-n \beta)}{\pi \Gamma(n-n \beta)} \\
\times \int_{0}^{z}\left(z^{\alpha}-u^{\alpha}\right)^{-n \beta+n-\lambda} u^{\alpha(n \beta+\lambda-n)-1 R L} D_{u}^{n(\beta-\alpha)}\left[E_{\alpha}\left(\tau u^{\alpha \beta}\right)\right] d u,
\end{gathered}
$$

where $z \in \mathbb{C}$ and $B$ is beta function.

Proof. The proof is immediately followed by replacing $\gamma$ with 1 in Theorem 3 .

Corollary 5. Let $\alpha, \beta, \lambda, \gamma, \tau \in \mathbb{C}$ and $\Re(\alpha)>0, \Re(\gamma)>0$ with $\Re(\lambda)<1$, we have:

$$
\begin{gathered}
M_{\alpha, \gamma}^{\beta, \lambda}\left(\tau z^{\alpha}\right)=\frac{\alpha \sin (\pi \lambda) B(1-\lambda, n-n \beta)}{\pi \Gamma(n-n \beta) \Gamma \alpha n+\gamma-n \beta} \\
\times \int_{0}^{z}\left(z^{\alpha}-u^{\alpha}\right)^{-n \beta+n-\lambda} u^{\alpha(n \beta+\lambda-n)-\gamma} \int_{0}^{u}(u-x)^{\alpha n+\gamma-n \beta-1} E_{\alpha, \gamma}\left(\tau x^{\alpha \beta}\right) d x d u,
\end{gathered}
$$

$z \in \mathbb{C}$.

Proof. Here, we have $\Re(1-\gamma)<0$; hence, the fractional diffintegral that occurs in the Equation (31) is an integral component. Thus, we obtain:

$$
{ }_{0}^{R L} D_{u}^{\alpha n+\gamma-n \beta-1}\left[E_{\alpha, \gamma}\left(\tau u^{\alpha \beta}\right)\right]=\frac{1}{\Gamma \alpha n+\gamma-n \beta} \int_{0}^{u}(u-x)^{\alpha n+\gamma-n \beta-1} E_{\alpha, \gamma}\left(\tau x^{\alpha \beta}\right) d x,
$$

and substitute this into Equation (31) to find:

$$
\begin{gathered}
M_{\alpha, \gamma}^{\beta, \lambda}\left(\tau z^{\alpha}\right)=\frac{\alpha \sin (\pi \lambda) B(1-\lambda, n-n \beta)}{\pi \Gamma(n-n \beta)} \\
\times \int_{0}^{z}\left(z^{\alpha}-u^{\alpha}\right)^{-n \beta+n-\lambda} u^{\alpha(n \beta+\lambda-n)-\gamma}\left[\frac{1}{\Gamma \alpha n+\gamma-n \beta} \int_{0}^{u}(u-x)^{\alpha n+\gamma-n \beta-1} E_{\alpha, \gamma}\left(\tau x^{\alpha \beta}\right) d x\right] d u,
\end{gathered}
$$

which gives the required proof.

Theorem 4. The Mittag-Leffler Four-Parameter function in Equation (16) can be given as an integral transformation of the following form:

$$
M_{\alpha, \gamma}^{\beta, \lambda}\left(\tau z^{\alpha}\right)=\frac{\alpha \sin (\pi \lambda) B(1-\lambda, n-n \beta)}{\pi \Gamma(n-n \beta) \Gamma \alpha n+\gamma-n \beta} \int_{0}^{z} \Omega_{\alpha, \gamma, \lambda}(x ; z) E_{\alpha, \gamma}\left(\tau x^{\alpha \beta}\right) d x, \quad z \in \mathbb{C},
$$

where $\alpha, \beta, \lambda, \gamma, \tau \in \mathbb{C}$ and $\Re(\alpha)>0, \Re(\gamma)>1$ with $\Re(\lambda)<1$, and $\Omega$ is a function defined as

$$
\Omega_{\alpha, \gamma, \lambda}(x ; z)=\int_{x}^{z}\left(z^{\alpha}-u^{\alpha}\right)^{-n \beta+n-\lambda} u^{\alpha(n \beta+\lambda-n)-\gamma}(u-x)^{\alpha n+\gamma-n \beta-1} d u .
$$

Proof. Fubini's theorem implies that the order of the integrals can be exchanged in Equation (33). We have $0 \leqslant u \leqslant z$ and $0 \leqslant x \leqslant u$, which is equivalent to $0 \leqslant x \leqslant z$ and $x \leqslant u \leqslant z$ after swapping. Thereby, we have (33):

$$
\begin{gathered}
M_{\alpha, \gamma}^{\beta, \lambda}\left(\tau z^{\alpha}\right)=\frac{\alpha \sin (\pi \lambda) B(1-\lambda, n-n \beta)}{\pi \Gamma(n-n \beta) \Gamma \alpha n+\gamma-n \beta} \\
\times \int_{0}^{z} \int_{0}^{u}\left(z^{\alpha}-u^{\alpha}\right)^{-n \beta+n-\lambda} u^{\alpha(n \beta+\lambda-n)-\gamma}(u-x)^{\alpha n+\gamma-n \beta-1} E_{\alpha, \gamma}\left(\tau u^{\alpha \beta}\right) d x d u
\end{gathered}
$$




$$
\begin{gathered}
=\frac{\alpha \sin (\pi \lambda) B(1-\lambda, n-n \beta)}{\pi \Gamma(n-n \beta) \Gamma \alpha n+\gamma-n \beta} \\
\times \int_{0}^{z} \int_{x}^{z}\left(z^{\alpha}-u^{\alpha}\right)^{-n \beta+n-\lambda} u^{\alpha(n \beta+\lambda-n)-\gamma}(u-x)^{\alpha n+\gamma-n \beta-1} E_{\alpha, \gamma}\left(\tau u^{\alpha \beta}\right) d u d x \\
=\frac{\alpha \sin (\pi \lambda) B(1-\lambda, n-n \beta)}{\pi \Gamma(n-n \beta) \Gamma \alpha n+\gamma-n \beta} \\
\times \int_{0}^{z} E_{\alpha, \gamma}\left(\tau u^{\alpha \beta}\right) \int_{x}^{z}\left(z^{\alpha}-u^{\alpha}\right)^{-n \beta+n-\lambda} u^{\alpha(n \beta+\lambda-n)-\gamma}(u-x)^{\alpha n+\gamma-n \beta-1} d u d x \\
=\frac{\alpha \sin (\pi \lambda) B(1-\lambda, n-n \beta)}{\pi \Gamma(n-n \beta) \Gamma \alpha n+\gamma-n \beta} \int_{0}^{z} E_{\alpha, \gamma}\left(\tau x^{\alpha \beta}\right) \Omega_{\alpha, \gamma, \lambda}(x ; z) d x,
\end{gathered}
$$

as required.

\section{Conclusions}

The study uses Riemann-Liouville fractional calculus to establish new links between the Mittag-Leffler functions of one to four parameters. Most probably, those findings can be extended in the future to simplify certain important physical models that utilize four parameters or more of Mittag-Leffler functions, or to offer more productive mathematical models for these functions, as the original Mittag-Leffler function is increasingly popular and studied in greater detail.

Author Contributions: Conceptualization, F.G., H.F.A.-J., and O.B.; methodology, F.G., H.F.A.-J., and O.B.; investigation, F.G., H.F.A.-J., and O.B.; resources, F.G., H.F.A.-J., and O.B.; data curation, F.G., H.F.A.-J., and O.B.; writing-original draft preparation, F.G., H.F.A.-J., and O.B.; writing-review and editing, F.G., H.F.A.-J., and O.B.; supervision, F.G., H.F.A.-J., and O.B.; project administration, F.G., H.F.A.-J., and O.B. All authors read and agreed to the published version of the manuscript.

Funding: This research received no external funding.

Institutional Review Board Statement: Not applicable.

Informed Consent Statement: Not applicable.

Data Availability Statement: Not applicable.

Conflicts of Interest: The authors declare no conflict of interest.

\section{References}

1. Miller, K.S.; Ross, B. An Introduction to the Fractional Calculus and Fractional Differential Equations; Wiley: New York, NY, USA, 1993.

2. Oldham, K.B.; Spanier, J. The Fractional Calculus; Academic Press: San Diego, CA, USA, 1974.

3. Samko, S.G.; Kilbasamd, A.A.; Marichev, O.I. Fractional Integrals and Derivatives: Theory and Applications; Original edited in Russian, Nauka i Tekhnika, Minsk, 1987; Taylor and Francis: London, UK, 2002.

4. Hilfer, R. (Ed.) Applications of Fractional Calculus in Physics; World Scientific: Singapore, 2000.

5. Kilbas, A.A.; Srivastava, H.M.; Trujillo, J.J. Theory and Applications of Fractional Differential Equations; Elsevier: Amsterdam, The Netherlands, 2006.

6. Podlubny, I. Fractional Differential Equations; Academic Press: San Diego, CA, USA, 1999.

7. Srivastava, H.M.; Fernandez, A.; Baleanu, D. Some New Fractional-Calculus Connections between Mittag-Leffler Functions. Mathematics 2019, 7, 485. [CrossRef]

8. Atangana, A.; Baleanu, D. New fractional derivatives with nonlocal and non-singular kernel, Theory and application to heat transfer model. Therm. Sci. 2016, 20, 763-769. [CrossRef]

9. Caputo, M.; Fabrizio, M. A new Definition of Fractional Derivative without Singular Kernel. Prog. Fract. Differ. Appl. 2015, 1, 73-85. 
10. Çetinkaya, A.; Kiymaz, I.O.; Agarwal, P.; Agarwal, R. A comparative study on generating function relations for generalized hypergeometric functions via generalized fractional operators. Adv. Differ. Equ. 2018, 2018, 156. [CrossRef]

11. Özarslan, M.A.; Ustaoğlu, C. Some Incomplete Hypergeometric Functions and Incomplete Riemann-Liouville Fractional Integral Operators. Mathematics 2019, 7, 483. [CrossRef]

12. Özarslan, M.A.; Ustaoğlu, C. Incomplete Caputo fractional derivative operators. Adv. Differ. Equ. 2018, 2018, 209. [CrossRef]

13. Ghanim, F.; Al-Janaby, H.F. Inclusion and Convolution Features of Univalent Meromorphic Functions Correlating with MittagLeffler Function. Filomat 2020, 34, 2141-2150. [CrossRef]

14. Ghanim, F.; Al-Janaby, H.F. Some Analytical Merits of Kummer-Type Function Associated with Mittag-Leffler Parameters. Arab. J. Basic Appl. Sci. 2021, 28, 255-263. [CrossRef]

15. Ghanim, F.; Bendak, S.; Hawarneh, A.A. Fractional Calculus Operator Involving Mittag- Leffler- Confluent Hypergeometric Functions. 2021, preprint.

16. Oros, G.I. Applications of Inequalities in the Complex Plane Associated with Confluent Hypergeometric Function. Symmetry 2021, 13, 259. [CrossRef]

17. Oros, G.I. Study on New Integral Operators Defined Using Confluent Hypergeometric Function. Adv. Differ. Equ. 2021, 1, 1-11.

18. Haubold, H.J.; Mathai, A.M.; Saxena, R.K. Mittag-Leffler functions and their applications. J. Appl. Math. 2011, $2011,298628$. [CrossRef]

19. Paneva-Konovska, J. Differential and integral relations in the class of multi-index Mittag-Leffler functions. Fract. Calc. Appl. Anal. 2018, 21, 254-265. [CrossRef]

20. Mainardi, F.; Gorenflo, R. On Mittag-Leffler-type functions in fractional evolution processes. J. Comput. Appl. Math. 2000, 118, 283-299. [CrossRef]

21. Mathai, A.M.; Haubold, H.J. Mittag-Leffler functions and fractional calculus. In Special Functions for Applied Scientists; Springer: New York, NY, USA, 2008; pp. 79-134.

22. Srivastava, H.M. On an extension of the Mittag-Leffler function. Yokohama Math. J. 1968, 16, 77-88.

23. Srivastava, H.M. Some families of Mittag-Leffler type functions and associated operators of fractional calculus (survey). TWMS J. Pure Appl. Math. 2016, 7, 123-145.

24. Bazighifan, O.; Dassios, I. Riccati Technique and Asymptotic Behavior of Fourth-Order Advanced Differential Equations. Mathematics 2020, 8, 590. [CrossRef]

25. Mittag-Leffler, G.M. Sur la nouvelle fonction $E_{\alpha}(x)$. C. R. Acad. Sci. Paris 1903, 137, 554-558.

26. Mittag-Leffler, G.M. Sur la repr'esentation analytique d;une branche uniforme d'une fonction monogene: Cinquieme note. Acta Math. 1905, 29, 101-181. [CrossRef]

27. Wiman, A. ÜBer den fundamentalsatz in der theorie der funcktionen $E_{\alpha}(x)$. Acta Math. 1905, 29, 191-201. [CrossRef]

28. Wiman, A. ÜBer die nullstellen der funktionen $E_{\alpha}(x)$. Acta Math. 1905, 29, 217-234. [CrossRef]

29. Baleanu, D.; Guvenc, Z.B.; Machado, J.A.T. New Trends in Nanotechnology and Fractional Calculus Applications; Springer: Dordrecht, The Netherlands; Heidelberg, Germany; London, UK; New York, NY, USA, 2010.

30. Baleanu, D.; Wu, G.C.; Zeng, S.D. Chaos analysis and asymptotic stability of generalized Caputo fractional differential equations. Chaos Solit. Fractals 2017, 102, 99-105. [CrossRef]

31. Esen, A.; Sulaiman, T.A.; Bulut, H.; Baskonus, H.M. Optical solitons and other solutions to the conformable space-time fractional Fokas-Lenells equation. Optik 2018, 167, 150-156. [CrossRef]

32. Veeresha, P.; Prakasha, D.G.; Baskonus, H.M. Solving smoking epidemic model of fractional order using a modified homotopy analysis transform method. Math. Sci. 2019, 13, 115-128. [CrossRef]

33. Prakasha, D.G.; Veeresha, P.; Baskonus, H.M. Analysis of the dynamics of hepatitis E virus using the Atangana-Baleanu fractional derivative. Eur. Phys. J. Plus 2019, 134, 241. [CrossRef]

34. Taneco-Hernández, M.A.; Morales-Delgado, V.F.; Gómez-Aguilar, J.F. Fractional Kuramoto—Sivashinsky equation with power law and stretched Mittag-Leffler kernel. Phys. A 2019, 527, 1210858. [CrossRef]

35. Wright, E.M. The asymptotic expansion of the generalized hypergeometric function. J. Lond. Math. Soc. 1935, 10, 286-293. [CrossRef]

36. Pillai, R.N. On Mittag-Leffler functions and related distributions. Ann. Inst. Statist. Math. 1990, 42, 157-161. [CrossRef]

37. Gorenflo, R.; Kilbas, A.A.; Mainardi, F.; Rogosin, S.V. Mittag-Leffler Functions, Related Topics and Applications; Springer: Berlin/Heidelberg, Germany, 2016.

38. Srivastava, H.M.; Tomovski, Ž. Fractional calculus with an integral operator containing a generalized Mittag-Leffler function in the kernel. J. Appl. Math. Comput. 2009, 211, 198-210. [CrossRef]

39. Macdonald, A.D. Properties of the confluent Hyper geometric function. J. Math. Phys. 1949, 28, 183-191. [CrossRef]

40. Davis, H.T. Tables of the Higher Mathematical Functions; The Principia Press: Bloomington, Indiana, 1933; Volume I.

41. Archibald, W.J. The complete solution of the differential equation for the confluent hypergeometric function. Lond. Edinb. Dublin Philos. Mag. J. Sci. 1938, 26, 415-419. [CrossRef]

42. Bateman, H.; Erdélyi, A. Higher Transcendental Functions; McGraw Hill: New York, NY, USA; Toronto, ON, Canada; London, UK, 1955; Volume 3.

43. Buschman, R.G. Convolution equations with generalized Laguerre polynomial kernels. SIAM Rev. 1964, 6, 166-167. [CrossRef] 
44. Khalendekar, P.R. On a convolution trans form involving generalized Laguerre polynomial as its kernel. J. Math. Pures Appl. 1965, 44, 195-197.

45. Prabhakar, T.R. A singular integral equation with a generalized Mittag-Leffler function in the kernel. Yokohama Math. J. 1971, 19, 7-15.

46. Rusia, K.C. An integral equation involving generalized Laguerre polynomial. Math. Jpn. 1966, 11, 15-18.

47. Bazighifan, O. On the oscillation of certain fourth-order differential equations with p-Laplacian like operator. Appl. Math. Comput. 2020, 386, 125475. [CrossRef]

48. Bazighifan, O.; Postolache, M. Improved Conditions for Oscillation of Functional Nonlinear Differential Equations. Mathematics 2020, 8, 552. [CrossRef]

49. Prabhakar, T.R. Two singular integral equations involving con fluent hypergeometric functions. Proc. Camb. Phil. Soc. 1969, 66, 71-89. [CrossRef]

50. Ghanim, F.; Al-Janaby, H.F. An analytical study on Mittag-Leffler-confluent hypergeometric functions with fractional integral operator. Math. Methods Appl. Sci. 2021, 44, 3605-3614. [CrossRef]

51. Srivastava, K.N. A class of integral equations involving Laguerre polynomials as Sur la representation analytique dune branche uniforme dune function kernel. Proc. Edinb. Math. Soc. 1966, 15, 33-36. [CrossRef]

52. Srivastava, K.N. On integral equations involving Whittaker's function. Proc. Glasg. Math. Assoc. 1966, 7, 125-127. [CrossRef]

53. Keiper, J.B. Fractional Calculus and Its Relationship to Riemann's Zeta Function. Master's Thesis, Ohio State University, Columbus, $\mathrm{OH}, \mathrm{USA}, 1975 ; 37 \mathrm{p}$. 